\title{
ANGLE OF INTERNAL FRICTION OF THE GRANULAR MATERIAL AND A SIMPLE TRANSIENT PHENOMENON
}

\author{
By Takeo Mogami*
}

\section{Synopsis}

In the first part of this paper, relationships between the angle of internal friction and the void in cases of axial symmetry and plane strain were derived by the theory of mechanics of granular material due to the present author.

With available data of shearing test on sand, the validity of these formulae mentioned above was examined, the results of which were satisfactory.

In the second part, experimental and theoretical study about the compression of one layered assemblage of steel balls randomly placed in a horizontal flat box was reported.

The phenomenon of spreading of compression area was explained fairly well by the theory and the observed values of pushing force of a wall showed good coincidence with those calculated by the theory. The theory presented by the author is based on statistical considerations following the same line of thought as in the previous paper.

\section{Introduction}

In the previous paper ${ }^{1}$, the author presented a statistical theory of mechanics of granular material in which the mechanical behaviour of the material at the stage of failure is discussed as an application of the general theory. He derived a relationship between the angle of internal friction and the void ratio in case when $\sigma_{3}=0$.

In the former part of this paper, similar relationships for axial symmetric and plane strain cases will be discussed. Further, experimental results and their theoretical considerations of the uni-axial compression of a one layered assemblage of steel balls will be presented.

The mechanics of granular material, taking the special character of grains into account, has been the main problem of the author since about thirty years ago. However, its difficulty

* Professor, University of Tokyo has overwhelmed him, so that he could get only little result about the matter. His experiences taught him that the study should be performed on the simplest cases of the simplest problem. Hence the experiments about the compression of the assemblage of steel balls of one layer were planned.

\section{Internal Angle of Friction}

In the previous paper, a relationship between the angle of internal friction and the void ratio was presented, that is

$$
\sin \phi=\frac{k}{1+e}, \quad k=a \text { constant }
$$

This expression was derived with the assumption $\sigma_{3}=0$, that is, the plane stress case, so that this is not always useful for problems. The formulae suitable to the cases which are seen in the usual testing procedures of soil will now be deduced.

The author is afraid if he gave an impression that his theory concerns only with the two dimensional problems, because it was applied to such a problem, therefore, he wants to give some remarks. No three dimensional experimental evidences have not yet been available until now and the author cannot apply his theory to three dimensional problems with confirmed experimental facts.

In two dimensional failure problem, experiments showed that the deviation of void ratio is zero at the moment of failure which was the basis of his failure theory, but similar experimental evidence has not been known for three dimensional cases.

However, the author believes that it is not so unreasonable to extend this experimental evidence to three dimensional cases and to adopt it as a basic assumption to such problems.

Denoting the three principal stresses by $\sigma_{1}$, $\sigma_{2}, \sigma_{3}$, the following identy is obvious,

$$
\left.\sigma_{1}=\frac{\sigma_{1}+\sigma_{2}+\sigma_{3}}{3}-\frac{\sigma_{3}-\sigma_{1}}{3}+\frac{\sigma_{1}-\sigma_{2}}{3}\right)
$$




$$
\left.\begin{array}{l}
\sigma_{2}=\frac{\sigma_{1}+\sigma_{2}+\sigma_{3}}{3}-\frac{\sigma_{1}-\sigma_{2}}{3}+\frac{\sigma_{2}-\sigma_{3}}{3} \\
\sigma_{3}=\frac{\sigma_{1}+\sigma_{2}+\sigma_{3}}{3}-\frac{\sigma_{2}-\sigma_{3}}{3}+\frac{\sigma_{3}-\sigma_{1}}{3}
\end{array}\right\}
$$

The increment of work done, $\Delta \mathrm{A}$, by three principal stresses on an element of volume is written down as

$$
\Delta A=-\sigma_{1} \epsilon_{1}-\sigma_{2} \epsilon_{2}-\sigma_{3} \epsilon_{3}
$$

where $\epsilon_{1}, \epsilon_{2}, \epsilon_{3}$ are increments of principal strains.

If we write

$$
\begin{aligned}
& \epsilon_{2}-\epsilon_{3}=-\Delta \gamma_{1}, \quad \frac{\sigma_{2}-\sigma_{3}}{2}=\tau_{1} \\
& \epsilon_{3}-\epsilon_{1}=-\Delta \gamma_{2}, \quad \frac{\sigma_{3}-\sigma_{1}}{2}=\tau_{2} \\
& \epsilon_{1}-\epsilon_{2}=-\Delta r_{3}, \quad \frac{\sigma_{1}-\sigma_{2}}{2}=\tau_{3} \\
& \frac{\sigma_{1}+\sigma_{2}+\sigma_{3}}{3}=p
\end{aligned}
$$

the increment of work done, $\Delta A$, can be rewritten as

$$
\Delta A=-\frac{p}{1+e} \Delta e+\frac{2}{3} \tau_{1} \Delta r_{1}+\frac{2}{3} \tau_{2} \Delta \gamma_{2}+\frac{2}{3} \tau_{3} \Delta \gamma_{3}
$$

where $e$ is the void ratio of the volume.

By the author's theory, presented in the previous paper, we can write

$$
\begin{aligned}
& \Delta A \equiv \Delta S=-\frac{p}{1+e} \Delta e+\frac{2}{3} \tau_{1} \Delta \gamma_{1}+\frac{2}{3} \tau_{2} \Delta \gamma_{2} \\
& +\frac{2}{3} \tau_{3} \Delta_{3}
\end{aligned}
$$

where

$$
\begin{aligned}
S=\frac{K}{N} & \log W \\
\log W & =C-e N \log e+N(1+e) \log (1+e) \\
& -\frac{N}{2} \frac{s}{e(1+e)}^{2)}
\end{aligned}
$$

$N=$ total number of grains in the volume

$C=a$ constant which does not depend on $e$ and $s$ $s=$ deviation of void ratio in the volume $K=a$ constant

\section{A) Axial symmetry case}

Here we can assume that $\sigma_{2}=\sigma_{3}$ and $\epsilon_{2}=\epsilon_{3}$, hence we have

$$
\begin{gathered}
\tau_{3}=\frac{\sigma_{1}-\sigma_{2}}{2}=-\tau_{2} \\
r_{1}=0, \epsilon_{3}-\epsilon_{1}=-\Delta \gamma_{2}=\Delta r_{3} \\
\text { and } \frac{\Delta e}{1+e}=\epsilon_{1}+2 \epsilon_{3}
\end{gathered}
$$

The increment of the work done is written down in this case as

$$
\Delta A=-\frac{p}{1+e} \Delta e+\frac{\sigma_{1}-\sigma_{3}}{3} \Delta r_{3}
$$

Following the similar way to the previous paper, we assume, introducing a coefficient $k_{3}$, that

$$
\log W=F\left(e-k_{3} r_{3}\right)
$$

The functional form $F$ is determined by the condition that $s=0$ for $r_{3}=0$. This condition is the extended one which was acertained by experiments in two dimensional cases, and about this assumption a remark was given before.

With the aid of the equations (6) and (8), and the above assumption, we can derive

$$
\begin{aligned}
& p=\frac{\sigma_{1}+2 \sigma_{3}}{3}=-K(1+e) \log w \\
& \frac{\sigma_{1}-\sigma_{3}}{3}=-K k_{3} \log w
\end{aligned}
$$

where

$$
w=\frac{1+e-k_{3} r_{3}}{e-k_{3} r_{3}}
$$

Then we have

$$
\left.\begin{array}{l}
\sigma_{1}+2 \sigma_{3}=-3 K(1+e) \log w \\
\sigma_{1}-\sigma_{3}=-3 K k_{3} \log w
\end{array}\right\}
$$

and

$$
\left.\begin{array}{l}
\sigma_{1}=-K\left(1+e+2 k_{3}\right) \log w \\
\sigma_{3}=-K\left(1+e-k_{3}\right) \log w
\end{array}\right\}
$$

Finally we have

$$
\sin \phi=\frac{\sigma_{1}-\sigma_{3}}{\sigma_{1}+\sigma_{3}}=\frac{3 k_{3}}{2(1+e)+k_{3}}
$$

The validity of this equation is examined by experimental data of triaxial compression by several authors.

Experimental data of void ratios and the angles of internal friction are inserted in the above equation and the constancy of the coefficient $k_{3}$ was examined, the results are shown in the following table 1.

These data were supplied from Winterkorn's paper $^{3)}$ except Inoue's data.

This table shows that the constancy of the value $k_{3}$ is fairly good. It can be seen that the constancy of the value $k$ in (1) is also good, which is a little curious.

\section{B) Plane strain case}

The plane strain is understood by the condition $\epsilon_{3}=0$. From equations (4), we have 
Table 1.

\begin{tabular}{c|c|r|r|r|r}
\hline$e$ & $\sin \phi$ & $k_{3}($ in (13)) & $\begin{array}{r}\left(k_{3}-\bar{k}_{3}\right) / \bar{k}_{3} \\
(\%)\end{array}$ & $k($ in (1)) & $\begin{array}{r}(k-\bar{k}) / \bar{k} \\
(\%)\end{array}$ \\
\hline 0.65 & 0.649 & 0.911 & 2.1 & 1.071 & 0.9 \\
0.70 & 0.624 & 0.893 & 0.0 & 1.061 & 0.0 \\
0.75 & 0.599 & 0.873 & -2.1 & $\frac{1.049}{\bar{k}=1.060}$ & -1.1 \\
& & $\overline{\bar{k}_{3}=0.892}$ & &
\end{tabular}

(D.W. Taylor; Fundamentals of Soil Mechanics)

\begin{tabular}{c|r|r|r|r|r}
\hline$e$ & $\sin \phi$ & $k_{3}(\operatorname{in}(13))$ & $\begin{array}{c}\left(k_{3}-\bar{k}_{3}\right) / \bar{k}_{3} \\
(\%)\end{array}$ & $k(\operatorname{in}(1))$ & \multicolumn{1}{c}{$\begin{array}{c}(k-\bar{k}) / \bar{k} \\
(\%)\end{array}$} \\
\hline 0.562 & 0.713 & 0.974 & 9.2 & 1.114 & \multicolumn{1}{c}{5.0} \\
0.695 & 0.631 & 0.903 & 1.2 & 1.069 & 0.8 \\
0.755 & 0.596 & 0.870 & -2.5 & 1.046 & -1.4 \\
0.886 & 0.537 & 0.821 & -8.0 & 1.013 & -4.5 \\
& & \multicolumn{1}{c}{$\bar{k}_{3}=0.892$} & & $\bar{k}=1.061$ & \\
\hline
\end{tabular}

(De Beer, E.; Neuere Erkenntnisse über den Bruchwiderstand Kohäsionloser Böden unter Flachgründungen, Sym. on Soil Mechanics, T.H. Aachen 1961)

\begin{tabular}{c|c|r|r|r|r}
\hline$e$ & $\sin \phi$ & $k_{3}(\operatorname{in}(13))$ & $\begin{array}{c}\left(k_{3}-\bar{k}_{3}\right) / \bar{k}_{3} \\
(\%)\end{array}$ & $k(\operatorname{in}(1))$ & $\begin{array}{c}(k-\bar{k}) / \bar{k} \\
(\%)\end{array}$ \\
\hline 0.640 & 0.653 & 0.913 & 4.9 & 1.071 & \multicolumn{1}{c}{3.3} \\
0.695 & 0.613 & 0.863 & -0.8 & 1.025 & -1.0 \\
0.754 & 0.577 & 0.835 & -4.1 & $\frac{1.012}{2.0}$ & -2.2 \\
& & $\bar{k}_{3}=0.870$ & & $\bar{k}=1.036$ & \\
\hline
\end{tabular}

(Nash, K.L., The Shearing Resistance of a fine Closely Graded Sand, Proc. 3rd Int. Conf. on Soil Mech. and Found. Eng. Zürich, 1953)

\begin{tabular}{c|c|r|r|r|r}
\hline$e$ & $\sin \phi$ & $k_{3}(\operatorname{in}(13))$ & $\begin{array}{r}\left(k_{3}-\bar{k}_{3}\right) / \bar{k}_{3} \\
(\%)\end{array}$ & $k(\operatorname{in}(1))$ & \multicolumn{1}{c}{$\begin{array}{c}(k-\bar{k}) \bar{k} \\
(\%)\end{array}$} \\
\hline 0.667 & 0.637 & 0.898 & 4.0 & 1.062 & 2.6 \\
0.725 & 0.612 & 0.884 & 2.6 & 1.051 & 1.5 \\
0.851 & 0.536 & 0.805 & -6.8 & 0.992 & -4.1 \\
& & $\bar{k}_{3}=0.862$ & & $\bar{k}=1.035$ & \\
\hline
\end{tabular}

(Bjerrum, L., Kingstad, S., and Kummenije, O., The Shear Strength of a Fine Sand. Proc. 5th Int. Conf. on Soil Mech. and Found. Eng., Paris 1961)

\begin{tabular}{c|c|r|r|r|r}
\hline$e$ & $\sin \phi$ & $k_{3}(\operatorname{in}(13))$ & $\begin{array}{r}\left(k_{3}-\bar{k}_{3}\right) / \vec{k}_{3} \\
(\%)\end{array}$ & $k(\operatorname{in}(1))$ & $\begin{array}{c}(\bar{k}-\bar{k}) / \bar{k} \\
(\%)\end{array}$ \\
\hline 0.60 & 0.568 & 0.747 & 1.1 & 0.909 & 0.4 \\
0.70 & 0.531 & 0.731 & -1.1 & 0.902 & -0.4 \\
& & $\overline{\bar{k}_{3}=0.739}$ & & $\bar{k}=0.905$ & \\
\hline
\end{tabular}

(Rutledge, P.C., Soil Mechanics Fact Finding Survey,

U.S. Army Corps of Engineers, Vicksburg, Miss. April 1947)

\begin{tabular}{c|c|r|r|r|c}
\hline$e$ & $\sin \phi$ & $k_{3}(\operatorname{in}(13))$ & $\begin{array}{c}\left(k_{3}-\bar{k}_{3}\right) / \bar{k}_{3} \\
(\%)\end{array}$ & $k(\operatorname{in}(1))$ & $\begin{array}{c}(k-\bar{k}) / \bar{k} \\
(\%)\end{array}$ \\
\hline 0.470 & 0.683 & 0.866 & 6.2 & 1.004 & 3.7 \\
0.667 & 0.559 & 0.764 & -6.0 & 0.932 & -3.7 \\
& & $\frac{0 . k_{3}=0.815}{\bar{k}=0.968}$ & & \\
\hline
\end{tabular}

(Bishop, A.W., A Large Shear Box for Testing Sands and Gravels., Proc. 2nd Int. Conf. on Soil Mech. and Found. Eng., Rotterdam 1948)

\begin{tabular}{c|r|r|r|r|r}
\hline$e$ & $\sin \phi$ & $k_{3}(\operatorname{in}(13))$ & $\begin{array}{r}\left(k_{3}-\bar{k}_{3}\right) / \bar{k}_{3} \\
(\%)\end{array}$ & $k(\operatorname{in}(1))$ & $\begin{array}{c}(k-\bar{k}) / \bar{k} \\
(\%)\end{array}$ \\
\hline 0.68 & 0.632 & 0.897 & -0.8 & 1.062 & -1.4 \\
0.75 & 0.619 & 0.911 & 0.8 & 1.082 & 0.7 \\
0.80 & 0.602 & 0.904 & 0.0 & 1.084 & 0.7 \\
& & $\overline{k_{3}=0.904}$ & & $\bar{k}=1.077$ & \\
\hline
\end{tabular}

(Inouye, H., Thesis)

\begin{tabular}{c|c|r|r|r|r}
\hline$e$ & $\sin \phi$ & $k_{3}(\operatorname{in}(13))$ & $\begin{array}{c}\left(k_{3}-\vec{k}_{3}\right) \vec{k}_{3} \\
(\%)\end{array}$ & $k($ in $(1))$ & $\begin{array}{c}(k-\bar{k}) / \bar{k} \\
(\%)\end{array}$ \\
\hline 0.67 & 0.681 & 0.981 & -1.5 & 1.138 & -3.3 \\
0.76 & 0.657 & 0.987 & -0.8 & 1.106 & 6.1 \\
0.87 & 0.641 & 1.016 & 2.1 & 1.199 & 1.8 \\
& & $k_{3}=0.995$ & & $\bar{k}=1.177$ & \\
\hline
\end{tabular}

(Inouye, H., Thesis)

\begin{tabular}{c|r|r|r|r|c}
\hline$e$ & $\sin \phi$ & $k_{3}(\operatorname{in}(13))$ & $\begin{array}{r}\left(k_{3}-\bar{k}_{3}\right) / \bar{k}_{3} \\
(\%)\end{array}$ & $k(\operatorname{in}(1))$ & $\begin{array}{c}(k-\bar{k}) / \bar{k} \\
(\%)\end{array}$ \\
\hline 0.80 & 0.675 & 1.045 & 0.7 & 1.265 & 2.4 \\
0.88 & 0.655 & 1.050 & 1.1 & 1.232 & 0.2 \\
0.90 & 0.635 & 1.020 & -1.7 & $\frac{1.207}{2}$ & 2.2 \\
& & $\overline{\bar{k}_{3}=1.038}$ & & $\bar{k}=1.235$ & \\
\hline
\end{tabular}

(Inouye, H., Thesis)

\begin{tabular}{c|r|r|r|r|r}
\hline$e$ & $\sin \phi$ & $k_{3}(\operatorname{in}(13))$ & $\begin{array}{r}\left(k_{3}-\bar{k}_{3}\right) / \bar{k}_{3} \\
(\%)\end{array}$ & $k(\operatorname{in}(1))$ & $\begin{array}{c}(k-\bar{k}) / \bar{k} \\
(\%)\end{array}$ \\
\hline 0.85 & 0.683 & 1.091 & -1.3 & 1.263 & 2.3 \\
0.94 & 0.662 & 1.099 & 0.6 & 1.289 & 0.2 \\
1.03 & 0.651 & 1.125 & 1.8 & 1.321 & 2.3 \\
& & $\bar{k}_{3}=1.105$ & & $\bar{k}=1.291$ & \\
\hline
\end{tabular}

(Inouye, H., Thesis)

$$
\begin{aligned}
& \epsilon_{1}=\Delta r_{2}=\frac{1}{2}\left(\frac{\Delta e}{1+e}-\Delta r_{3}\right) \\
& \epsilon_{2}=-\Delta r_{1}=\frac{1}{2}\left(\frac{\Delta e}{1+e}+\Delta r_{3}\right) \\
& \Delta r_{3}=-\left(\Delta r_{1}+\Delta r_{2}\right)
\end{aligned}
$$

Then the increment of work done, $\Delta A$, can be written as

$$
\Delta A=-\frac{\sigma_{1}+\sigma_{2}}{2(1+e)} \Delta e+\frac{1}{3}\left(\sigma_{1}-\sigma_{2}\right) \Delta \gamma_{3} \cdots
$$

As in the previous paragraph, we can get

where

$$
\begin{aligned}
& \sigma_{1}+\sigma_{2}=-2(1+e) K \log w \\
& \sigma_{1}-\sigma_{2}=-K k_{3} \log w
\end{aligned}
$$

$$
w=\frac{1+e-k_{3} r_{3}}{e-k_{3} r_{3}}
$$

Hence we have

$$
\left.\begin{array}{l}
\sigma_{1}=-\frac{1}{2}\left\{2(1+e)+3 k_{3}\right\} K \log w \\
\sigma_{2}=-\frac{1}{2}\left\{2(1+e)-3 k_{3}\right\} K \log w
\end{array}\right\}
$$

Finally we have

$$
\sin \phi=\frac{\sigma_{1}-\sigma_{2}}{\sigma_{1}+\sigma_{2}}=\frac{3 k_{3}}{2(1+e)}
$$

The expression (18) is examined by the experimental data for plane strain by Whitman and Luscher ${ }^{4}$. (Table 2) The constancy of $k_{3}$ is very good.

C) Discussion on the Direct Shear Test

The stress or strain condition in sample under direct shear is not so simple, so that above 
Table 2.

\begin{tabular}{c|l|l|r|r}
\hline$e$ & $\phi$ & $\sin \phi$ & $\boldsymbol{k}_{3}(\operatorname{in}(18))$ & $\begin{array}{c}\left(\boldsymbol{k}_{3}-\overline{\boldsymbol{k}}_{3}\right) / \overline{\boldsymbol{k}}_{3} \\
(\%)\end{array}$ \\
\hline 0.491 & $42^{\circ}$ & 0.669 & 0.665 & 1.5 \\
0.500 & $41^{\circ} 12^{\prime}$ & 0.659 & 0.659 & 0.6 \\
0.519 & $40^{\circ}$ & 0.643 & 0.651 & -0.6 \\
0.540 & $39^{\circ}$ & 0.629 & 0.646 & -1.4 \\
& & & $\overline{\boldsymbol{k}_{3}=0.655}$ & \\
\hline
\end{tabular}

Table 3.

\begin{tabular}{c|c|c|r|r|r|c}
\hline$e$ & $\tan \phi$ & $\sin \phi$ & $k_{3}(\operatorname{in}(13))$ & $\begin{array}{c}\left(k_{3}-\bar{k}_{3}\right) / \bar{k}_{3} \\
(\%)\end{array}$ & $k_{3}(\operatorname{in}(18))$ & $\begin{array}{c}\left(k_{3}-\bar{k}_{3}\right) / \bar{k}_{3} \\
(\%)\end{array}$ \\
\hline 0.56 & 0.649 & 0.544 & 0.691 & 7.9 & 0.566 & 5.6 \\
0.59 & 0.512 & 0.512 & 0.654 & 2.2 & 0.542 & 1.1 \\
0.62 & 0.554 & 0.485 & 0.625 & -2.3 & 0.524 & -2.2 \\
0.64 & 0.539 & 0.474 & 0.615 & -3.9 & 0.518 & -3.4 \\
0.66 & 0.531 & 0.469 & 0.615 & -3.9 & 0.519 & -3.2 \\
& & & $\overline{\bar{k}_{3}=0.640}$ & & $\overline{\bar{k}_{3}=0.536}$ & \\
\hline
\end{tabular}

(D.W. Taylor, Fundamentals of Soil Mechanics)

\begin{tabular}{c|c|c|r|r|r|r}
\hline$e$ & $\tan \phi$ & $\sin \phi$ & $k_{3}(\operatorname{in}(13))$ & $\begin{array}{r}\left(k_{3}-\bar{k}_{3}\right) / \bar{k}_{3} \\
(\%)\end{array}$ & $k_{3}(\operatorname{in}(18))$ & $\begin{array}{c}\left(k_{3}-\bar{k}_{3}\right) / \bar{k}_{3} \\
(\%)\end{array}$ \\
\hline 0.65 & 0.980 & 0.700 & 1.004 & 4.0 & 0.770 & 1.4 \\
0.73 & 0.900 & 0.669 & 0.993 & 2.9 & 0.771 & 1.6 \\
0.87 & 0.756 & 0.603 & 0.941 & -2.5 & 0.752 & -0.9 \\
0.94 & 0.705 & 0.576 & 0.922 & -4.5 & 0.744 & -2.0 \\
& & & $\bar{k}_{3}=0.965$ & & $\bar{k}_{3}=0.759$ & \\
\hline
\end{tabular}

(Inouye, H. Thesis)

\begin{tabular}{|c|c|c|c|c|c|c|}
\hline$e$ & $\tan \phi$ & $\sin \phi$ & $k_{3}(\mathrm{in}(13))$ & $\begin{array}{c}\left(k_{3}-\bar{k}_{3}\right) \bar{k}_{3} \\
(\%)\end{array}$ & $k_{3}(\mathrm{in}(18))$ & $\begin{array}{c}\left(k_{3}-\bar{k}_{3}\right) / \vec{k}_{3} \\
(\%)\end{array}$ \\
\hline 0.76 & 1.626 & 0.852 & 1.396 & 8.4 & 1.000 & 4.5 \\
\hline 0.82 & 1.386 & 0.811 & 1.349 & 4.7 & 0.984 & 2.8 \\
\hline 0.88 & 1.212 & 0.771 & 1.301 & 1.0 & 0.964 & 0.7 \\
\hline 0.94 & 1.058 & 0.727 & 1.241 & -3.7 & 0.940 & -1.8 \\
\hline \multirow[t]{2}{*}{1.00} & \multirow[t]{2}{*}{0.908} & \multirow[t]{2}{*}{0.672} & 1.155 & \multirow[t]{2}{*}{-10.3} & 0.896 & \multirow[t]{2}{*}{-6.4} \\
\hline & & & $\bar{k}_{3}=1.288$ & & $\bar{k}_{3}=0.957$ & \\
\hline
\end{tabular}

theories cannot be applied directly to the results of direct shear of sands.

Nevertheless the author tried to examine the applicability of the equations (13) and (18) to this case. (Table 3 )

Both $k_{3} \mathrm{~s}$ in (13) and in (18) are fairly constant in every set of experimental data, however, it seems that the constancy of $k_{3}$ in (18) is a little better than that of $k_{3}$ in (13).

\section{Compression of an Assemblage of Balls}

\section{1) Experimental evidences}

Steel balls of equal size $($ diameter $=0.23 \mathrm{~cm}$ ) were placed in one layer on the glass bottom plate of a horizontal flat box $(25 \mathrm{~cm} \times 15 \mathrm{~cm})$ as randomly as possible and one side wall $A B$ was pushed in. The box is shown in the figure 1. Experiments were carried out for three initial void ratios, namely $0.85,0.57$ and 0.39 .

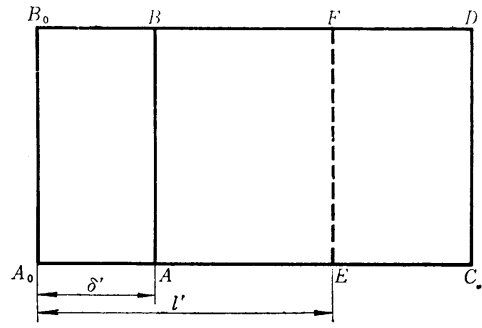

Fig. 1 The flat box used in the experiments

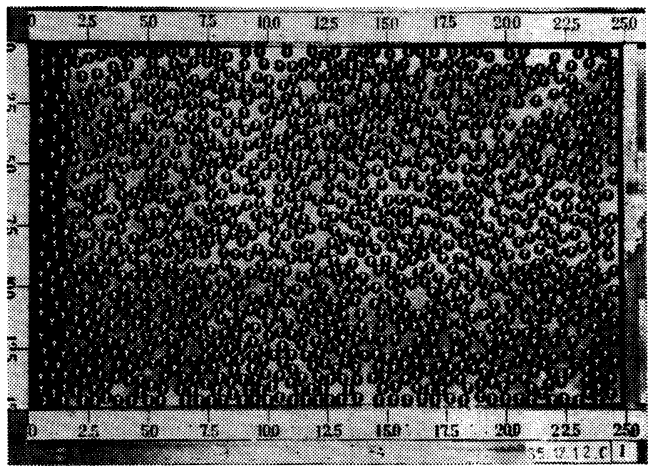

Fig. 2

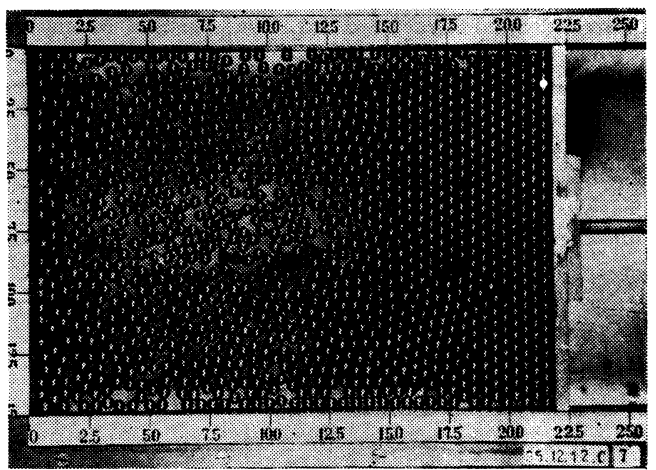

Fig. 3

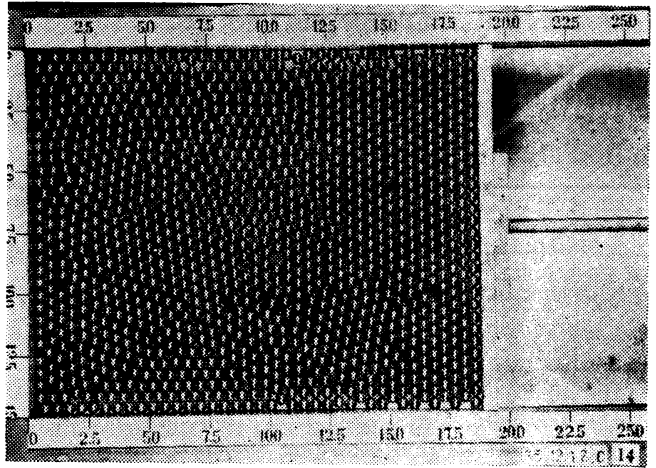

Fig. 4

Photo-cells were placed under the bottom of the box, the light emitted from electric lamps which were located above the box was received by the photo-cells. The amount of light received by a photo-cell is proportional to the space 
between balls, so that the void ratio of an area can be measured by a photo-cell placed beneath this area.

When one side wall $A_{0} B_{0}$ is pushed in to the position $A B$, assemblage of balls close to this side wall is compressed and becomes much crowded, this compressed area spreads towards the opposite wall of the box by the displacement of the wall (Figure 2 4).

The void ratio of an area located at a distance 1' (Figure 1) remains constant until the displacement of the side wall reaches $\delta^{\prime}$ and after that the void ratio begins to decrease (Figure 5). It indicates that the compression spreads by a distance $1^{\prime}$ when the displacement of the

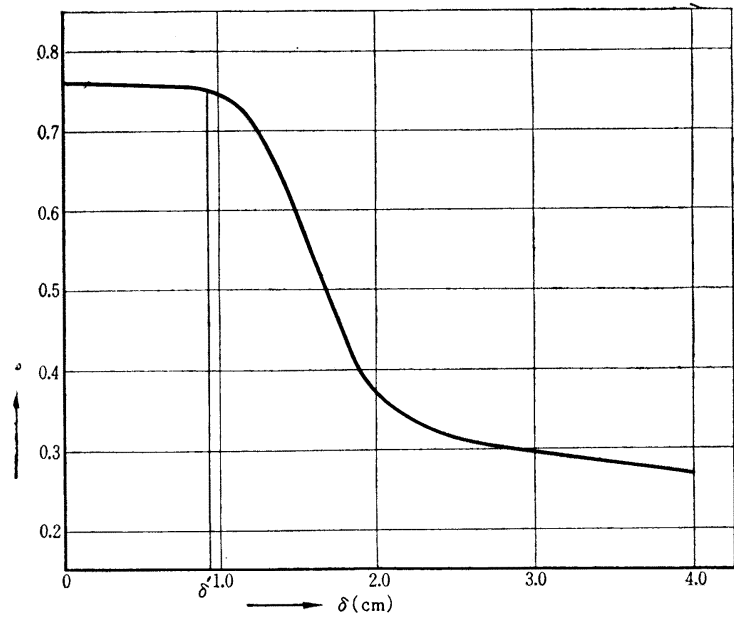

Fig. 5 The change in void ratio at one place with the displacement of a side wall. wall attains $\delta^{\prime}$.

On the other hand, some balls were coloured yellow to make clear the trace of motion of them, it showed that they move almost on straight lines along the longer sides.

The force to move the wall was measured and plotted against the displacement of the wall. (Figure 6). These plottings were made by averaging the data obtained by 60 experiments. The force is almost proportional to the displacement when the initial void ratio is larger, but it curves remarkably in case of smaller void ratio.

\section{2) Theoretical considerations}

(a) Spreading of compressed area

$A_{0} B_{0}$ in the figure 1 is the initial position of the movable side wall, $\mathrm{AB}$ is the position of the wall at one moment and EF is the position of the end of compressed area at the same moment.

As shown in the former paragraphs, steel balls move almost straight, hence if the width of the box is divided into channels of width of the diameter of a steel ball, it can be said that all balls move along a channel to which it belongs (Figure 7).

If every channel is divided into $n$ cells, each of which can contain one ball, in every channel, $r$ cells contain balls and the other $n-r$ cells are empty. If steel balls in the box are distributed uniformly, the void ratio of one channel coincides with the void ratio of

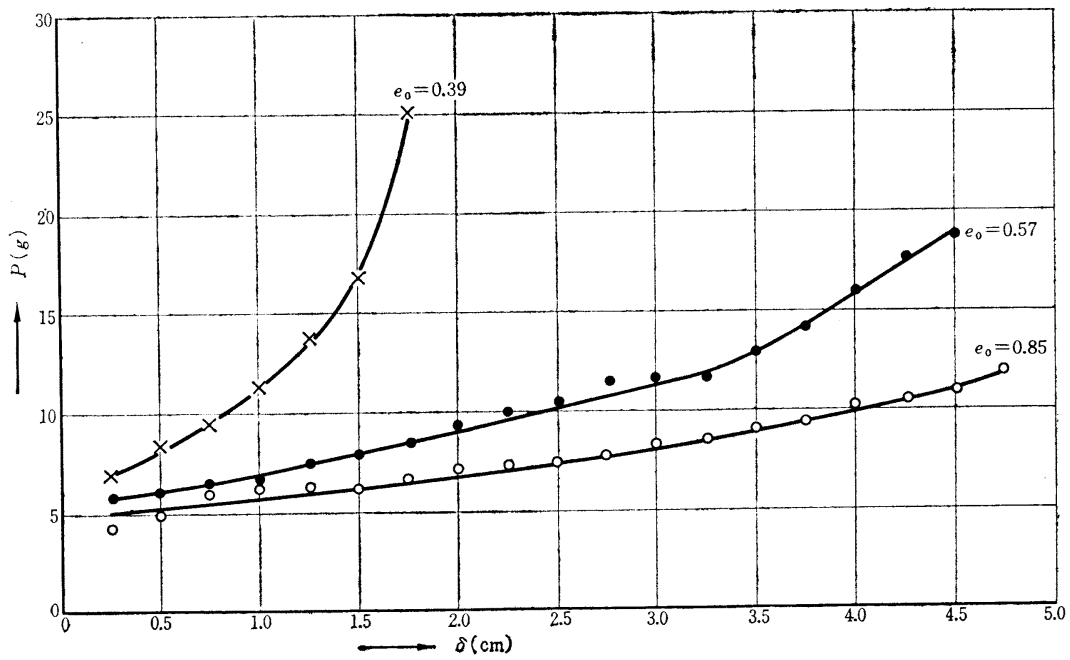

Fig. 6 Relationship between the pushing force and displacement of the wall. 


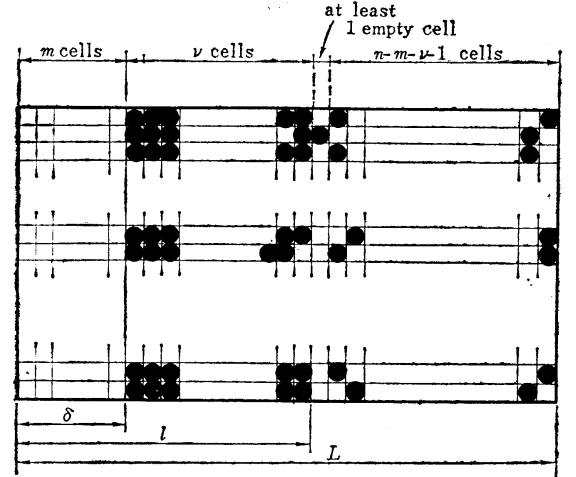

Fig. 7 Schematic view of the arrangement of balls in the box.

the total area, so that

$$
\frac{n-r}{r}=e_{0} \quad \therefore \frac{r}{n}=p_{0}=\frac{1}{1+e_{0}}
$$

where $e_{0}$ is the initial void ratio of the total area.

Take out an arbitrary channel, if the displacement $\delta$ of the wall is equivalent to $m$ cells and if the compression phenomenon spreads to $E$, which is equivalent to $\nu$ cells, next one cell is empty and other $n-m-\nu-1$ cells contain $r-\nu$ balls (Figure 7). The situation of other channels are not always same as this one channel, so that we have to make average of all channels.

The number $N_{\nu}$ of ways of arrangement of balls as shown in the figure 7 is the number of ways of putting $r-\nu$ balls in $n-m-\nu-1$ cells, so that

$$
N_{\nu}=\left(\begin{array}{c}
n-m-\nu-1 \\
r-\nu
\end{array}\right)
$$

With the aid of the formula

$$
\begin{aligned}
\sum_{r=1}^{n}\left(\begin{array}{c}
a+r-1 \\
a
\end{array}\right) & =\frac{(a+1)(a+2) \cdots(a+n)}{n !}-1 \\
& =\left(\begin{array}{c}
a+n \\
a
\end{array}\right)-1=\left(\begin{array}{c}
a+n \\
n
\end{array}\right)-1
\end{aligned}
$$

we can show that

$$
\sum_{\nu=0}^{r}\left(\begin{array}{c}
n-m-\nu-1 \\
r-\nu
\end{array}\right)=\left(\begin{array}{c}
n-m \\
r
\end{array}\right)
$$

Hence we can get the probability $P_{\nu}$ that the balls are arranged in the way shown in the figure 7 as

$$
P_{\nu}=\left(\begin{array}{c}
n-m-\nu-1 \\
r-\nu
\end{array}\right) /\left(\begin{array}{c}
n-m \\
r
\end{array}\right)
$$

With this probability, we can calculate the average value of $\nu$. Let us write it by the same letter for the sake of simplicity, the number of balls arranged in one array contacting with each other.

$$
\begin{aligned}
& E\{\nu\}=\sum_{\nu=1}^{r} P_{\nu} \cdot \nu= \\
& \text { using the same letter for simplicity's }
\end{aligned}
$$

To get the concrete expression of the value of $E\{\nu\}$, some calculations are necessary.

Putting $r-\nu=N, k=n-m-r-1$, the following calculations are carried out.

$$
\begin{aligned}
& \sum_{\nu=0}^{r}\left(\begin{array}{c}
n-m-\nu-1 \\
r-\nu
\end{array}\right) \nu \\
& =\sum_{N=r}^{0}\left(\begin{array}{c}
n-m-r+N-1 \\
N
\end{array}\right)(r-N) \\
& =\sum_{N=0}^{r}\left(\begin{array}{c}
k+N \\
k
\end{array}\right)(r-N)
\end{aligned}
$$

By the formula (21), we get

$$
\begin{aligned}
\sum_{N=0}^{r}\left(\begin{array}{c}
k+N \\
k
\end{array}\right) r & =r\left[\left(\begin{array}{c}
k+r+1 \\
k+1
\end{array}\right)-1\right] \\
& =r\left[\left(\begin{array}{c}
n-m \\
n-m-r
\end{array}\right)-1\right] \cdots \cdots
\end{aligned}
$$

With the aid of the formula

$$
\left(\begin{array}{c}
n+1 \\
x
\end{array}\right)=\left(\begin{array}{c}
n \\
x
\end{array}\right)+\left(\begin{array}{c}
n \\
x-1
\end{array}\right)
$$

we can write down

$$
\begin{aligned}
& \left(\begin{array}{c}
k+1 \\
k
\end{array}\right)=\left(\begin{array}{c}
k \\
k
\end{array}\right)+\left(\begin{array}{c}
k \\
k-1
\end{array}\right) \\
& \left(\begin{array}{c}
k+2 \\
k
\end{array}\right)=\left(\begin{array}{c}
k+1 \\
k
\end{array}\right)+\left(\begin{array}{l}
k+1 \\
k-1
\end{array}\right) \\
& \cdots \cdots \cdots \cdots \cdots \cdots \cdots \cdots \cdots \cdots \cdots \cdots \cdots \cdots \\
& \left(\begin{array}{c}
k+r \\
k
\end{array}\right)=\left(\begin{array}{c}
k+r-1 \\
k
\end{array}\right)+\left(\begin{array}{c}
k+r-1 \\
k-1
\end{array}\right)
\end{aligned}
$$

Multiplying $1,2, \cdots, r$ to each of above expressions respectively and adding term by term, we have

$$
\begin{aligned}
r\left(\begin{array}{c}
k+r \\
r
\end{array}\right) & =\sum_{N=1}^{r} N\left(\begin{array}{c}
k+N-1 \\
N
\end{array}\right)+\sum_{N=r}^{r-1}\left(\begin{array}{c}
k+N \\
N
\end{array}\right)+1 \\
& =\sum_{N=1}^{r} N\left(\begin{array}{c}
k+N-1 \\
N
\end{array}\right)+\left(\begin{array}{c}
k+r \\
r-1
\end{array}\right) \cdots \cdots \cdots(28) \cdots \cdots\left(\begin{array}{c}
k+r-1 \\
r-1
\end{array}\right)
\end{aligned}
$$

Applying the expressions (26) and (29) to (25), we can get

$$
\begin{aligned}
E\{\nu\}= & {\left[r\left(\begin{array}{c}
k+r+1 \\
r
\end{array}\right)-r\left(\begin{array}{c}
k+r+1 \\
r
\end{array}\right)\right.} \\
& \left.+\left(\begin{array}{c}
k+r+1 \\
r-1
\end{array}\right)\right] /\left(\begin{array}{c}
n-m \\
r
\end{array}\right)=\frac{r}{n-m-r+1}=\nu
\end{aligned}
$$


If we multiply on both sides of the expression (30) the diameter $\phi$ of a steel ball, we have

$$
\begin{aligned}
& \frac{l-\delta}{L}=\frac{\nu \phi}{n \phi}=\frac{p_{0}}{n-m-r+1}=\frac{p_{0} \phi}{n \phi\left[1-p_{0}-\frac{\delta}{L}\right]} \\
& =\frac{p_{0}}{1-p_{0}} \frac{\phi}{L} \frac{1}{1-\frac{1}{1-p_{0}} \frac{\delta}{L}} \\
& \therefore l-\delta=\frac{\phi}{e_{0}}\left[1+\frac{1+e_{0}}{e_{0}} \frac{\delta}{L}\right. \\
& +\left(\frac{1+e_{0}}{e_{0}}\right)^{2}\left(\frac{\delta}{L}\right)^{2}+\cdots \cdots \cdot
\end{aligned}
$$

This expression shows that $l-\delta$ is linearly connected with $\delta$ if higher order terms in $\delta / L$ are neglected and the tangent of the angle of inclination is linearly related to $\left(1+e_{0}\right) / e_{0}{ }^{2}$. These two relations are certified by experiments as shown in the figure 8 and 9 , the exact validity of the expression (32) could not be certified by experiments, that is, the values which are calculated by the right hand terms of the expression (32) inserting given values of $\phi, e_{0}$, $L$ and $\delta$ do not coincide with the values of its left hand side term obtained by experiments.

But it may be said, the author believes, that the expression (32) would tell the truth of things about the matter though the numerical coincidence was not so exact, because as stated above the expression (32) can be brought to a much more satisfactory one, if it is rewritten as

$$
\begin{aligned}
\therefore l-\delta= & \frac{C \phi}{e_{0}}\left[1+\frac{1+e_{0}}{e_{0}} \frac{\delta}{L}\right. \\
& \left.+\left(\frac{1+e_{0}}{e_{0}}\right)^{2}\left(\frac{\delta}{L}\right)^{2}+\cdots \cdots\right] \ldots
\end{aligned}
$$

where $C$ is a constant.

\section{3) The relationship between the force and displacement}

As shown in the previous paragraph, when the side wall is pushed in, the compressed area spreads towards the opposite wall. In this case the balls which belong to the compressed area move together with the motion of the wall, whilst balls in un-compressed area remain indifferent. Hence the frictional force between balls and the bottom plate of the box would act only on balls in the compressed area, then this force may be proportional to $l-\delta$ and hence to $\delta$ according to the expression (32).

However, experimental evidence which is shown in the figure 6 tells us that the applied force is not proportional to the displacement of the wall especially when the initial void ratio is less.

In addition to the above said frictional force, there exist frictional forces which act between balls, their character is much more complicated than the frictional force between balls and the bottom plate.

But we can proceed the discussions along the same line of thought as that was adopted in the previous paper.

By use of the Stirling's formula

$$
\begin{aligned}
& \log (n-a) ! \fallingdotseq(n-a)\{\log (n-a)-1\} \\
& \log (r-b) ! \fallingdotseq(r-b)\{\log (r-b)-1\}
\end{aligned}
$$

if $n \gg a$ and $r \gg b$

$$
\begin{aligned}
\therefore \log \left(\begin{array}{c}
n-a \\
r-b
\end{array}\right) \fallingdotseq & (n-a) \log (n-a) \\
& -(r-b) \log (r-b) \\
& -(n-a-r+b) \log (n-a-r+b)
\end{aligned}
$$

Then we get

$$
\begin{aligned}
& \log \left(\begin{array}{c}
n-m-\nu-1 \\
r-1
\end{array}\right) \\
& \fallingdotseq(n-m-\nu-1) \log (n-m-\nu-1) \\
& \quad-(r-\nu) \log (r-\nu) \\
& \quad-(n-m-r-1) \log (n-m-r-1) \\
& \log \left(\begin{array}{c}
n-m \\
r
\end{array}\right) \fallingdotseq(n-m) \log (n-m)-r \log r \\
& \quad-(n-m-r) \log (n-m-r)
\end{aligned}
$$

Applying these formulae and the expression (30) which gives $\nu$, in that expression $\nu$ is written for average $\nu$, to the expression (23), we get

$$
\begin{aligned}
& \log P_{\nu} \fallingdotseq\left\{(n-m)-\frac{n-m+1}{n-m-r+1}\right\} \log \left\{n-m-\frac{n-m+1}{n-m-r+1}\right\} \\
& -r\left(1-\frac{1}{n-m-r+1}\right) \log \left\{r\left(1-\frac{1}{n-m-r+1}\right)\right\}-\{n-m-r-1\} \log \{n-m-r-1\} \\
& -(n-m) \log (n-m)+r \log r+(n-m-r) \log (n-m-r) \\
& =-\frac{n-m}{n-m-r} \log (n-m)+\frac{r}{n-m-r} \log r+\log (n-m-r)
\end{aligned}
$$


Then we have

$$
\begin{aligned}
\frac{d \log P_{\nu}}{d m}= & \frac{1}{n-m-r} \log (n-m)-\frac{n-m}{(n-m-r)^{2}} \log (n-m) \\
& +\frac{1}{n-m-r}+\frac{r}{(n-m-r)^{2}} \log r-\frac{1}{n-m-r} \\
= & \frac{-\left(1+e_{0}\right)}{n e_{0}{ }^{2}\left(1-\frac{1+e_{0}}{e_{0}} \frac{\delta}{L}\right)^{2}}\left[\log \left(1+e_{0}\right)+\log \left(1-\frac{\delta}{L}\right)\right] \\
\therefore \frac{d \log P_{\nu}}{d \delta}= & \frac{-\left(1+e_{0}\right)}{e_{0}{ }^{2} L\left(1-\frac{1+e_{0}}{e_{0}} \frac{\delta}{L}\right)^{2}}\left[\log \left(1+e_{0}\right)+\log \left(1-\frac{\delta}{L}\right)\right]
\end{aligned}
$$

The work done by the external force during the increment $d \delta$ of the displacement $\delta$ is $P d \delta$, and this energy is equal to the energy loss due to the frictional force between balls and the

bottom plate and the energy loss due to the change of state of arrangement of balls, which is proportional to $\left\{d \log P_{\nu} / d \delta\right\} d \delta$, hence we get

$$
P d \delta=\alpha_{0} \frac{1+e_{0}}{e_{0}{ }^{2}} \delta d \delta+\beta_{0} \frac{1}{L} \frac{1+e_{0}}{e_{0}{ }^{2}} \frac{\log \left(1+e_{0}\right)+\log \left(1-\frac{\delta}{L}\right)}{\left(1-\frac{1+e_{0}}{e_{0}{ }^{2}} \frac{\delta}{L}\right)^{2}} d \delta
$$

where, $\alpha_{0}, \beta_{0}$ are constants which give the distributing factors of energy loss into two kinds

of source of loss.

$$
P=\alpha_{0} \frac{1+e_{0}}{e_{0}{ }^{2}} \delta+\frac{\beta_{0}}{L} \frac{1+e_{0}}{e_{0}{ }^{2}} \frac{\log \left(1+e_{0}\right)+\log \left(1-\frac{\delta}{L}\right)}{\left(1-\frac{1+e_{0}}{e_{0}{ }^{2}} \frac{\delta}{L}\right)^{2}} .
$$

For the sake of simplicity, this expression is rewritten as

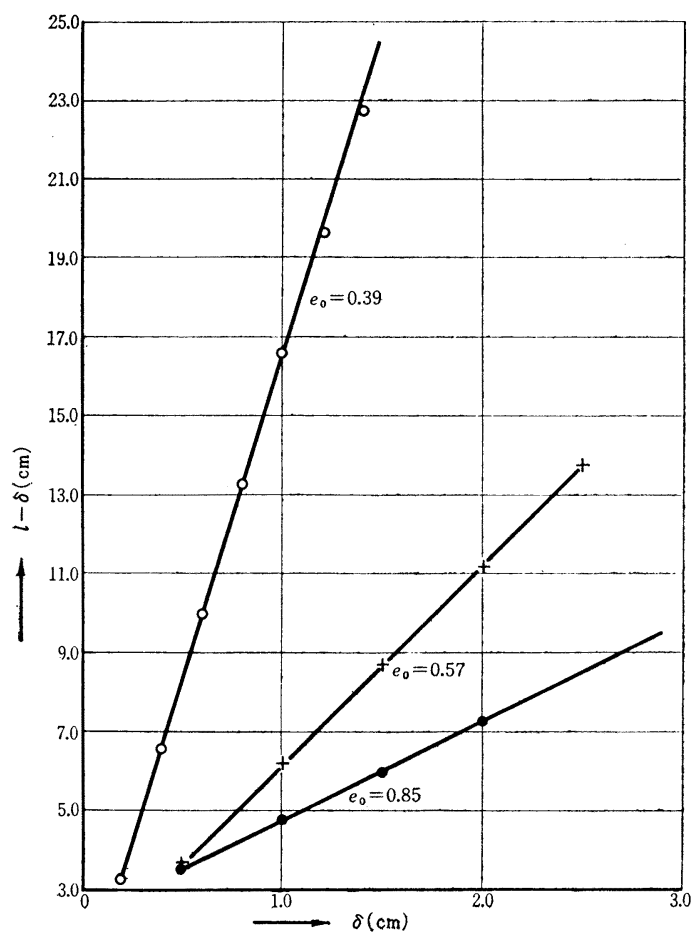

Fig. 8 Plotting explaining the validity of the equation (32)

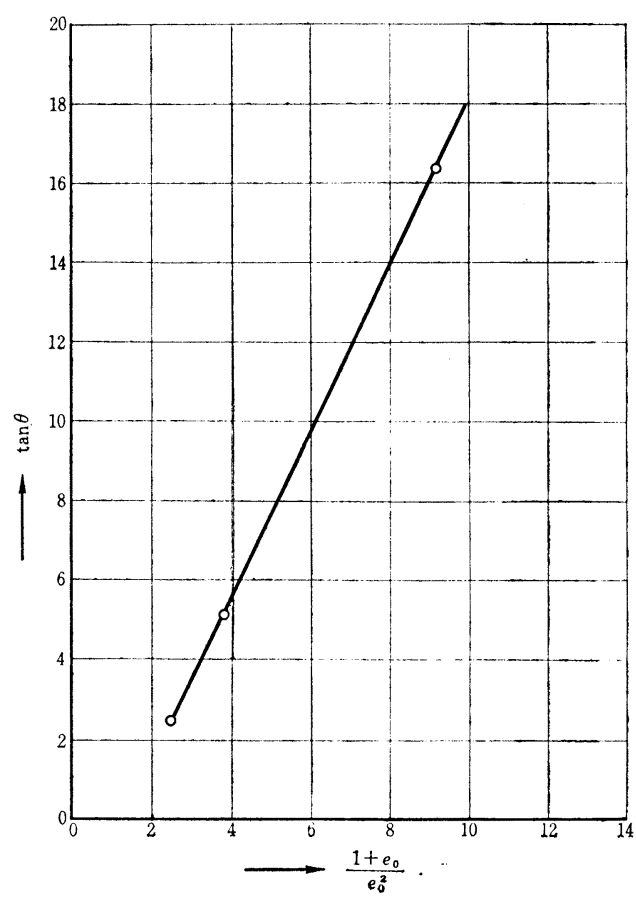

Fig. 9 Plotting explaining the validity of the equation (32) 


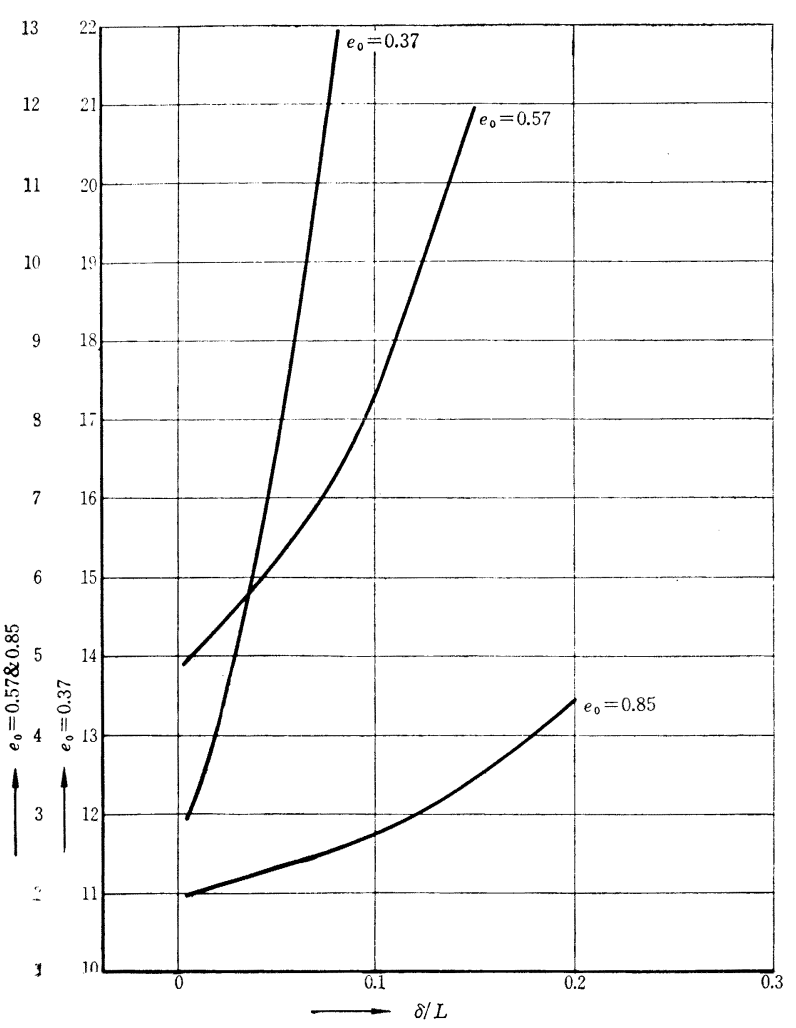

Fig. 10 Calculated values of

$$
\begin{gathered}
\frac{1+e_{0}}{e_{0}^{2}} \frac{1}{\left(1+\frac{1+e_{0}}{e_{0}} \frac{\delta}{L}\right)^{2}}\left[\log \left(1+e_{0}\right)+\log \left(1-\frac{\delta}{L}\right)\right] \\
P=\alpha_{0} I+\frac{\beta_{0}}{L} I I \ldots \ldots \ldots \ldots \ldots \ldots \ldots \ldots \ldots \ldots(42)
\end{gathered}
$$

where

$$
\begin{aligned}
& I=\frac{1+e_{0}}{e_{0}{ }^{2}} \delta \\
& I I=\frac{1+e_{0}}{e_{0}{ }^{2}} \frac{\log \left(1+e_{0}\right)+\log \left(1-\frac{\delta}{L}\right)}{\left(1-\frac{1+e_{0}}{e_{0}{ }^{2}} \frac{\delta}{L}\right)^{2}}
\end{aligned}
$$

The values of $I$ can be obtained by the figure 8 by giving the values of $e_{0}$ and $\delta$, whilst the values of $I I$ can be calculated for values of $e_{0}$ and $\delta / L$. Values of $I I$ are calculated and plotted against $\delta / L$ in the figure 10 .

With experimental data for two sets of $P$ and $\delta$ shown in the figure 6 , two equations for $\alpha_{0}$ and $\beta_{0} / L$ are obtained.

By this procedure we get the followings, for examples.

(i) $e_{0}=0.39$

For two values of $\delta=0.5 \mathrm{~cm}, 1.0 \mathrm{~cm}$, that is, $\delta / L=0.02,0.04$, values of $I$ are obtained from the figure $8, I=11.3,16.6$ respectively and values of $I I$ are calculated by the expression (43) as $I I=13.26$ and 15.35, hence we have with the aid of the figure 6 .

$$
\begin{aligned}
& 11.3 \alpha_{0}+13.26 \frac{\beta_{0}}{L}=8.2 \\
& 16.6 \alpha_{0}+15.35 \frac{\beta_{0}}{L}=11.2
\end{aligned}
$$

These equations give

$$
\alpha_{0}=0.485, \quad \frac{\beta_{0}}{L}=0.205
$$

Hence the equation (42) is completed for the initial void ratio $e_{0}=0.39$ as

$$
P=0.485 I+0.205 I I
$$

Inserting arbitrary value of $\delta$ we can calculate the force $P$ corresponding to this given displacement $\delta$, for examples

$$
\text { for } \begin{aligned}
\delta & =1.5 \mathrm{~cm}, \frac{\delta}{L}=0.06, \\
P & =0.485 \times 24.8+0.205 \times 18.25 \\
& =15.77
\end{aligned}
$$

for $\delta=1.25 \mathrm{~cm}, \frac{\delta}{L}=0.05$

$$
\begin{aligned}
P & =0.485 \times 20.4+0.205 \times 16.56 \\
& =13.28
\end{aligned}
$$

Observed values of force, that is, the forces which can be read on curves in the figure 6 are

$$
\begin{aligned}
P & =16.8 \mathrm{gm} \\
& =13.6 \mathrm{gm}
\end{aligned}
$$

respectively, hence the coincidence between theoretical and experimental values is very satisfactory.

(ii) $e_{0}=0.57$

In the similar manner mentioned above, using the observed values for $\delta=0.5 \mathrm{~cm}, 1.0 \mathrm{~cm}$, we obtain

$$
\begin{aligned}
\alpha_{0} & =0.023, \frac{\beta_{0}}{L}=1.118 \\
\therefore P & =0.023 I+1.118 I I \ldots
\end{aligned}
$$

With this equation the forces corresponding to the displacements of $2.5 \mathrm{~cm}$ and $1.5 \mathrm{~cm}$ were calculated as

for $\delta=2.5 \mathrm{~cm}$

$$
P=0.023 \times 13.8+1.118 \times 8.38=9.686
$$

for $\delta=1.5 \mathrm{~cm}$

$$
P=0.023 \times 8.7+1.118 \times 6.65=7.635
$$

Observed values are $10.4 \mathrm{gm}$ and $8.0 \mathrm{gm}$ 
which show good agreement.

(iii) $e_{0}=0.85$

With the observed data for $\delta=2 \mathrm{~cm}$ and 3 $\mathrm{cm}$, we have

$$
\alpha_{0}=-0.032, \frac{\beta_{0}}{L}=2.782
$$

Similar to the previous cases, we have

$$
\text { for } \alpha=2.5 \mathrm{~cm}
$$

$$
P=-8.5 \times 0.032+2.8 \times 2.782=7.518
$$

for $\alpha=1.5 \mathrm{~cm}$

$$
P=-3.8 \times 0.032+2.4 \times 2.782=6.557
$$

Observed values for these cases are respectively

$$
P=7.4 \mathrm{gm} \text { and } 6.4 \mathrm{gm}
$$

Hence the coincidence is also very good.

As it is shown in the above three examples $\alpha_{0}$ and $\frac{\beta_{0}}{L}$ change with the void ratio, $\alpha_{0}$ decreases and $\frac{\beta_{0}}{L}$ increases with the void ratio. Especially $\alpha_{0}$ decreases to the negative value, however, this negative value should be taken to be zero, the appearance of the negative value would be resulted from some causes in numerical computations. The values $\alpha_{0}, \frac{\beta_{0}}{L}$ are

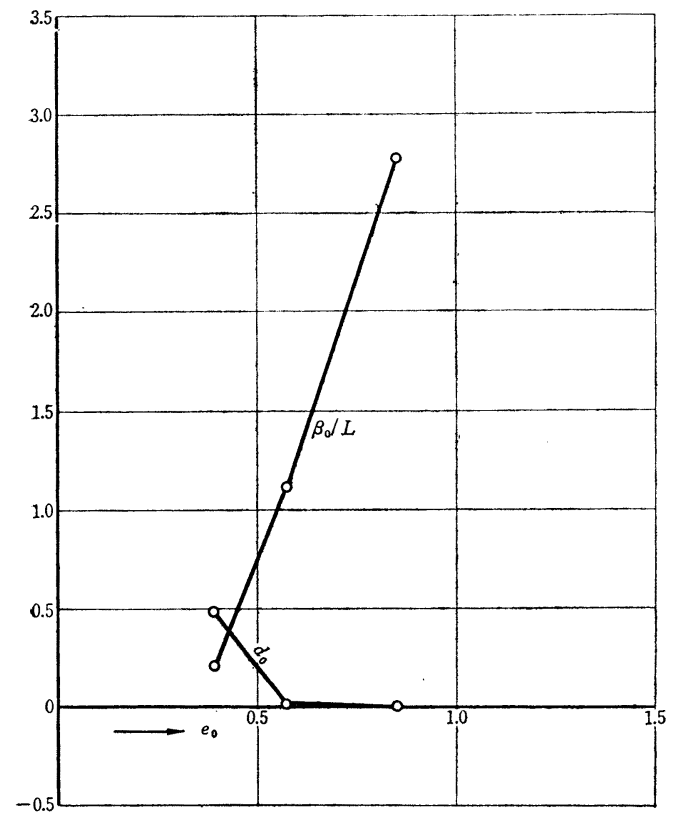

Fig. 11 Change of the coefficients $\alpha_{0}, \beta_{0} / L$ in the equation (27) with void ratio

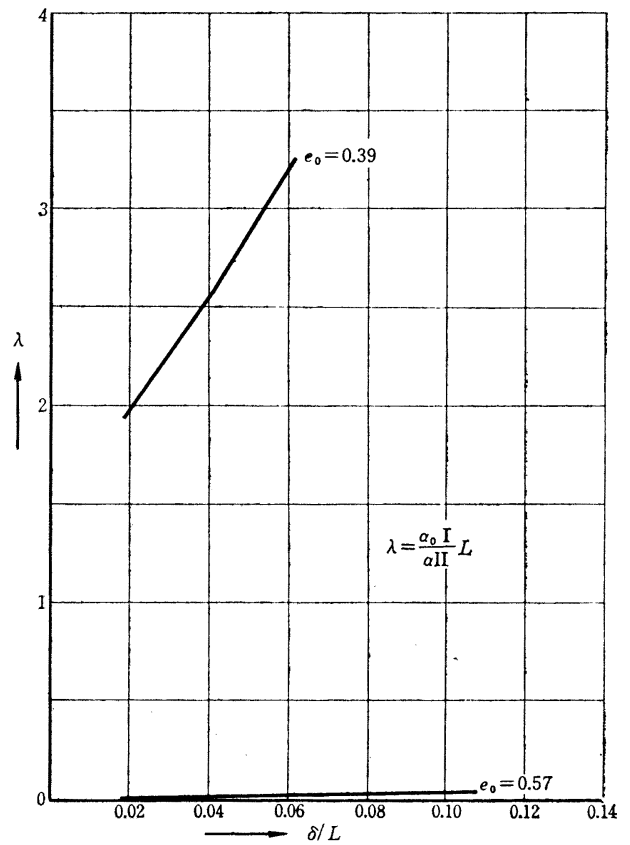

Fig. 12 Change of the ratio of two coefficients in the equation (27) with displacement

plotted against the initial void ratio in the figure 11. The degree of contribution of the two sources of energy loss is estimated by the ratio of $\alpha_{0} I$ to $\frac{\beta_{0}}{L} I I$, which is written as $\lambda$.

$$
\lambda=\frac{\alpha_{0} I}{\beta_{0} I I} L
$$

Value of $\lambda$ are shown in the figure 12. As shown by the curves in this figure, if the initial void ratio is smaller the contribution of the bottom friction is predominent, in looser cases bottom friction contributes only negligibly small amount of resistance.

\section{Reference}

1) T. Mogami, A statistical approach to the mechanics of granular material, Soil and Foundation Vol. V , No. 2, 1965. or A Statistical Theory of Mechanics of Granular Materials, Jorn. Faculty of Eng. Univ. of Tokyo, Ser. (B), Vol. 28, No. 2, 1965.

2) The sign of $\log W$ given in the previous paper was not correct. It is corrected in this paper.

3) Omar T. Farouki, Hans, F. Winterkorn; Mechanical properties of granular systems; Highway Research Record, Numbes 52, 1964

4) W.D. Liam Finn and H.K. Mittal, Shear Strength of Soil in a General Stress Field., ASTM Special Technical Publication No. 361, Laboratory Shear Testing of Soils, 1964

(Received Aug. 25, 1965) 